\title{
A NOTE ON THE MOMENTS OF THE NUMBER OF AXIS-CROSSINGS BY A STOCHASTIC PROCESS
}

\author{
BY M. R. LEADBETTER ${ }^{1}$
}

\section{Communicated by M. Kac, September 21, 1966}

1. Introduction. A general formula, for moments of arbitrary order of the number of upcrossings of a level $u$ by a stationary normal process in unit time, was obtained by Cramér and Leadbetter [1], using a combination of techniques due to $\mathrm{Kac}$ [3], and Ylvisaker [6]. Ylvisaker [7] has weakened the conditions of this result slightly by a proof which depends on interesting applications of martingale convergence theory and which may be applied also to nonstationary normal situations. In this note we give a somewhat different direct procedure, under the weakened conditions, for the calculation of these moments. This procedure gives an alternative to that of Ylvisaker [7] for normal processes, without the use of martingale theory, and may be also applied to nonnormal situations in the same way as the discussion in [4] for the first moment.

We shall here give the "counting procedure" used to obtain the number of upcrossings, sketching the derivation, and indicating the extension to nonnormal cases. A detailed proof along these lines (for the stationary normal case) will be given elsewhere (Cramér and Leadbetter [2]).

2. A general result. We shall consider a process $x(t)$ possessing, a.s., continuous sample functions and, for a given integer $k$, absolutely continuous $2 k$-dimensional distributions with corresponding densities of the form $f_{t_{1} \ldots t_{2 k}}\left(x_{1} \cdots x_{2 k}\right)$. There will be no loss of generality in considering the number $N$ of upcrossings of the zero level by $x(t)$ in $0 \leqq t \leqq 1$, which is a well-defined random variable (cf. [4]).

For $t=\left(t_{1} \cdots t_{k}\right)$ lying in the $k$-dimensional unit cube, let $m_{r}$ denote the unique integer such that $m_{r} / 2^{n} \leqq t_{r}<\left(m_{r}+1\right) / 2^{n}$. Write $E_{n}(t)$ for the $k$-dimensional cube whose sides are the intervals $\left[m_{r} / 2^{n},\left(m_{r}+1\right) / 2^{n}\right)$. For $\epsilon>0$, let $A_{n e}$ denote the set of all points $t$ in the unit cube such that for all $s=\left(s_{1} \cdots s_{k}\right) \in E_{n}(t)$, we have $\left|s_{i}-s_{j}\right|>\epsilon$ whenever $i \neq j$, and write $\lambda_{n \mathrm{e}}(t)$ for the characteristic function of the set $A_{n e}$. Finally let the random variable $\chi_{i, n}=1$ if $x\left(i / 2^{n}\right)<0<x\left[(i+1) / 2^{n}\right], \chi_{i, n}=0$ otherwise. The following lemma

1 This research was supported by the Army, Navy, Air Force and NASA under a contract administered by the Office of Naval Research. Reproduction in whole or in part is permitted for any purpose of the United States Government. 
gives the basic properties of the counting procedure used to obtain the factorial moments of $N$.

LEMma. Let $M_{n \epsilon}=\sum_{x_{i_{1}, n} \cdots x_{i_{k}, n}}^{\prime} \lambda_{n \epsilon}\left(i_{1} / 2^{n} \cdots i_{k} / 2^{n}\right)$, where the summation is extended over all ordered sets of distinct integers $i_{1} \cdots i_{k}$, $0 \leqq i_{r} \leqq 2^{n}-1$. Then, with probability one,

(i) $M_{n \in}$ is nondecreasing as $n$ increases or as $\epsilon$ decreases,

(ii) $\lim _{n \rightarrow \infty} \lim _{\epsilon \rightarrow 0} M_{n \varepsilon}=N(N-1) \cdots(N-k+1)$.

The proof of this lemma is accomplished by arguments extending those in [1, Part B]. From the monotonicity properties stated in (i) of the lemma it follows that the order of the $\epsilon$ and $n$-limits in (ii) may be interchanged. Hence, writing $M_{k}=\varepsilon N(N-1) \cdots(N-k+1)$, two applications of monotone convergence show that $M_{k}$ $=\lim _{\mathrm{e} \rightarrow 0} \lim _{n \rightarrow \infty} \& M_{n}$. From the definition of $M_{n \mathrm{e}}$ and a simple transformation of variables we thus have

$$
\begin{aligned}
M_{k}=\lim _{e \rightarrow 0} \lim _{n \rightarrow \infty} & \sum^{\prime} \lambda_{n e}\left(i_{1} / 2^{n} \cdots i_{k} / 2^{n}\right) \\
& \cdot P\left\{x_{i_{r}}<0<x_{i_{r}}+2^{-n} y_{i_{r}}, r=1,2, \cdots, k\right\}
\end{aligned}
$$

in which $x_{r}=x\left(r / 2^{n}\right)$ and $y_{r}=2^{n}\left(x_{r+1}-x_{r}\right)$. In fact, the only nonzero terms in the sum on the right correspond to integers $i_{1} \cdots i_{k}$ satisfying $\left|i_{r}-i_{s}\right|>1$ for $r \neq s$. For such integer sets, the random variables $x_{i_{1}} \cdots x_{i_{k}}, y_{i_{1}} \cdots y_{i_{k}}$ possess a joint density. Write $\psi_{n t \epsilon}\left(x_{1} \cdots, x_{k}\right.$, $\left.y_{1} \cdots y_{k}\right)$ to be equal to this joint density for all $t=\left(t_{1} \cdots t_{k}\right)$ of $A_{n e}$ lying in the cube $E_{n}\left(i_{1} / 2^{n} \cdots i_{k} / 2^{n}\right)$, and $\psi_{n \neq \mathrm{e}}=0$ outside such cubes. From (1) we then obtain the following result by straightforward calculation.

THEOREM. For the process $x(t)$ considered, the kth factorial moment of $N$ is

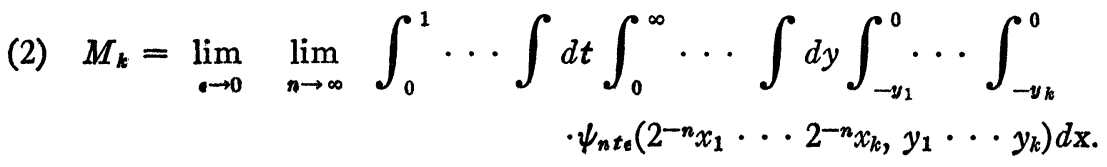

3. Normal processes and generalizations. It can easily be seen that the above assumptions are satisfied for a (separable) stationary normal process $x(t)$ whose covariance function has a finite second derivative at the origin. Further if $p_{t}(x, y)$ denotes the joint density for $x\left(t_{1}\right) \cdots x\left(t_{k}\right)$ and the quadratic mean derivatives $x^{\prime}\left(t_{1}\right) \cdots x^{\prime}\left(t_{k}\right)$, it can be shown (cf. [5]) by convergence of covariances that $\psi_{n t}\left(2^{-n} x_{1} \cdots 2^{-n} x_{k}, y_{1} \cdots y_{k}\right) \rightarrow p_{t}(0, y)$ as $n \rightarrow \infty$, for all $t$ in the 
region $D(\epsilon)=\lim _{n \rightarrow \infty} A_{n \epsilon}$. It can be shown by dominated convergence that the $n$-limit in (2) can be taken inside all the integral signs, and it is then an easy application of monotone convergence as $\epsilon \rightarrow 0$ to obtain the result:

$$
M_{k}=\int_{0}^{1} \cdots \int d t \int_{0}^{\infty} \cdots \int y_{1} \cdots v_{k} p t(0, y) d y \leqq \infty .
$$

Certain nonnormal processes may be treated in a similar way from (2) to obtain (3), (see, for example, the derivation of $\varepsilon N$ in [4] for the envelope of a stationary normal process.) In general we may obtain a result corresponding to that given in [4] for the mean. To that end write, for $t=\left(t_{1} \cdots t_{k}\right)$,

$$
g_{t, \tau}(\mathrm{x}, y)=\tau^{k} f_{t_{1} \cdots t_{k}, t_{1}+\tau \cdots t_{k}+\tau}\left(x_{1} \cdots x_{k}, x_{1}+\tau y_{1} \cdots x_{k}+\tau y_{k}\right) .
$$

That is $g_{t, \tau}$ is the joint density for the $x\left(t_{i}\right)$ and the incrementary ratios $\left(x_{t_{i}+\tau}-x_{t_{i}}\right) / \tau$. Then we have the following result.

Theorem. Consider points $t=\left(t_{1} \cdots t_{k}\right)$ such $t_{i} \neq t_{j}$ for $i \neq j$ and suppose that,

(i) $g_{t \tau}(x, y)$ is continuous in $(t, x)$ for each $y, \tau$,

(ii) For each $\epsilon>0, g_{t r}(x, y) \rightarrow p_{t}(x, y)$ as $\tau \rightarrow 0$ uniformly in $(t, x)$ for $t \in D(\epsilon)$ and each $y$.

(iii) For each $\epsilon>0$, there is a function $h_{\epsilon}(y)$ such that for $t \in D(\epsilon)$,

$$
g_{t, \tau}(x, y) \leqq h_{\epsilon}(y) \text { and } \int_{0}^{\infty} \cdots \int y_{1} \cdots y_{k} h_{\epsilon}(y) d y<\infty .
$$

Then (3) holds for the process $x(t)$.

Acknowledgments. I am very grateful to Professor Harald Cramér and to Dr. J. D. Cryer for their helpful comments and conversations concerning this and related problems.

\section{REFERENCES}

1. Harald Cramer and M. R. Leadbetter, The moments of the number of crossings of a level by a stationary normal process. Ann. Math. Statist. 36 (1965), 1656-1663.

2. - Stationary and related stochastic processes, Wiley, New York, (to appear).

3. $\mathrm{M}$. Kac, On the average number of real roots of a random algebraic equation Bull. Amer. Math. Soc. 49 (1943), 314-320.

4. M. R. Leadbetter, On crossings of levels and curves by a wide class of stochastic processes, Ann. Math. Statist. 37 (1966), 260-267.

5. M. R. Leadbetter and J. D. Cryer, On the mean number of curve crossings by non-stationary normal processes, Ann. Math. Statist. 36 (1965), 509-516. 
6. N. D. Ylvisaker, The expected number of zeros of a stationary Gaussian process, Ann. Math. Statist. 36 (1965), 1043-1046.

7. - On theorem of Cramer and Leadbetter, Ann. Math. Statist. 37 (1966), 682-685.

University of North Carolina and Research Triangle Institute 